\title{
Reconstruction of Patient-Specific Left Atrial Geometry From CMR Imaging
}

\author{
Soo-Kng Teo ${ }^{1 *}$, Xiaodan Zhao ${ }^{2}$, Ru-San Tan ${ }^{2,3}$, Liang Zhong ${ }^{2,3}$, Yi Su ${ }^{1}$ \\ ${ }^{1}$ Institute of High Performance Computing, A*STAR, Singapore \\ ${ }^{2}$ National Heart Centre Singapore, Singapore \\ ${ }^{3}$ Duke-NUS Medical School, National University of Singapore
}

\begin{abstract}
Clinical quantification of left atrial (LA) volumes is currently performed using either the biplane area-length method or the method of discs (Simpson's method) on 2-D cardiac images or $3 D$ echocardiography. However, these methods tend to underestimate LA volumes as compared to cardiovascular magnetic resonance (CMR) imaging.

In this paper, we propose a geometry-based reconstruction algorithm for computing the LA volume automatically for the entire cardiac cycle by combining information from both the short- and long-axis from CMR imaging. The inputs to our reconstruction algorithm are as follows: (i) a set of segmented short-axis contours and (ii) a set of segmented long-axis contours from the standard 2chamber and 4-chamber views. Our approach consists of a series of iterative steps where the most basal short-axis contour is projected in the atrial direction and subsequently morph to the patient-specific LA shape using the long-axis contours as guide. These series of morphing generate a left heart comprising both the LV and LA geometries with a planar basal surface. To reconstruct the LA cap, this planar basal surface is morphed into a hemisphere representing the closed surface of the LA using the long-axis contours as guide, thereby allowing us to reconstruct the closed LA shape and to calculate its volume.
\end{abstract}

\section{Introduction}

Left atrial (LA) dilatation has been associated with cardiac diseases and results from either the chronic volume and/or pressure overload of the LA. Studies have shown that LA dilation has been associated with the severity of left ventricular (LV) diastolic dysfunction [1] and it is also a recognized adverse prognostic marker in several cardiac diseases [2, 3].

Currently, clinical quantification of LA volumes is performed using either the biplane area-length method or the method of discs (Simpson's method) on 2-D echocardiography images based on the recommendations from the American Society of Echocardiography [4]. However, these methods tend to underestimate LA volumes as compared to cardiovascular magnetic resonance (CMR) imaging, an imaging modality that yields high-quality images of heart chambers, and is generally regarded as the gold standard for measuring LV volume and ejection fraction (EF). However, measuring LA volume from CMR using the above methods is timeconsuming as it requires the acquisition of additional images and additional analysis time [5, 6]. Furthermore, the LA volume computed using the biplane area-length method or the Simpson's method is based on 2D images and not the actual 3D geometry of the LA. This is because it is challenging to obtain realistic 3D LA geometry based on CMR images because of its complex anatomy and very thin myocardial wall [7].

In our approach, we proposed to overcome these challenges by using the radial basis function morphing algorithm to reconstruct the 3D LA geometry by combining information from both the long-axis and shortaxis from CMR. This reconstruction is performed iteratively by the projection and subsequent morphing of the most basal short-axis contours in the atrial direction, using the long-axis contours as a guide. The advantage of our approach is twofold: (i) Our approach relies on standard cardiac magnetic resonance imaging sequence that is used routinely for clinical examination. This is in contrast to other methods for computing LA volumes either through the use of multi-slice computed tomography or special MRI sequences and (ii) Computation of the LA volume is performed using the actual $3 \mathrm{D}$ reconstructed geometry. This is in contrast to conventional methods of using 2D measurements on the images (typically, using the 2-chamber and 4-chamber long-axis views) for volume measurement such as the biplane area-length and Simpson's methods.

The clinical utility of this approach is illustrated in a group of heart-failure (HF) patients. The current diagnostic of $\mathrm{HF}$ patients relays primarily on evaluation of the $\mathrm{LV}$ and its function. Impaired systolic (contractile) pump function 
identifies HF with reduced EF. However, $50 \%$ of HF have preserved EF (HFPEF), which is associated with increased heart muscle stiffness and inability of the heart to fill properly (diastolic dysfunction). Therefore, comprehensive assessment of both systolic and diastolic parameters in the LV and LA is needed to diagnosis HF, especially HFPEF to allows for the better characterization of the overall cardiac function for HF patients.

\section{Methods}

The overview of the method for the $3 \mathrm{D}$ geometrical reconstruction of the LA-LV models is briefly summarized as follows:

(i) CMR acquisition. Standard cine-MRI protocol is used in this work. This is an advantage as no additional requirement is needed for our method to work.

(ii) Image segmentation. The long-axis and short-axis contours of the LA and LV are extracted and used as the initial input for the ensuing algorithms.

(iii) Projection/Morphing of short-axis contour (optional). This is a set of iterative procedure where the most basal short-axis contour is projected in the atrial direction and subsequently morph to the patient-specific LA shape using the long-axis contours as guide. This step is required if the entire LA is not captured in the CMR imaging.

(iv) Morphing to reconstruct the LA-LV interface. This is to capture the non-planar mitral valve interface that separate the LV from the LA.

(v) Reconstruction/Morphing of the planar basal surface.

\subsection{CMR Acquisition}

All subjects underwent CMR scanning balanced turbo field echo (BTFE) sequence imaged on a 3.0 T Philips scanner (Ingenia, Philips Healthcare, Netherlands) with a dStream Torso coil (maximal number of channels 32). BTFE end-expiratory breath hold cine images were acquired in multi-planar short- and long-axis views. The CMR images have a spatial resolution of $1.5 \mathrm{~mm}$ in-plane and $8 \mathrm{~mm}$ out-of-plane, acquired in a single breath hold, with 30 temporal phases per cardiac cycle.

\subsection{Image Segmentation}

Image segmentation was performed on both the shortaxis and long-axis CMR images using the MR postprocessing software Medis "Qstrain” (Version 2.0, Medis Medical Imaging Systems, Leiden, the Netherlands). Manual delineation of the LA and LV contours were performed by an experienced radiographer at the enddiastole and end-systole frames. Segmentation at all subsequent frames was performed by the software utilising a feature tracking algorithm. For the short-axis, segmentation for the LV and LA includes all images. For the long-axis, segmentation on the LA and LV was performed on the 2-chamber $(2 \mathrm{CH})$ and 4-chamber $(4 \mathrm{CH})$ views.

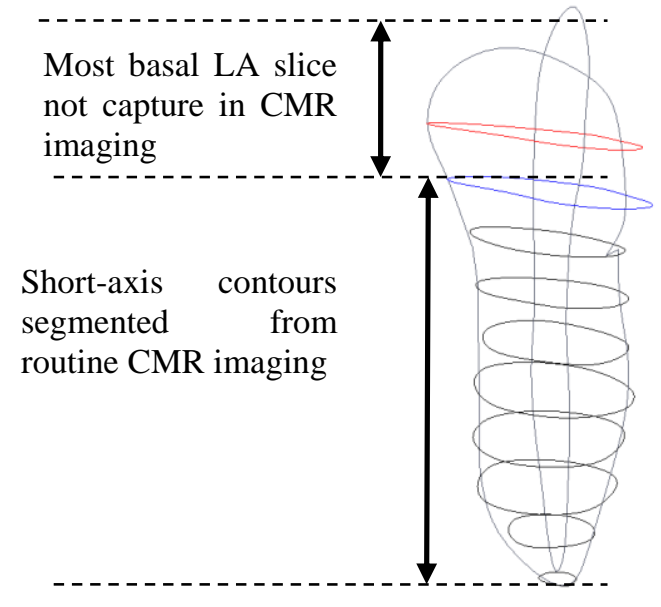

Figure 1. Projection and morphing of the most basal short-axis contours segmented from routine CMR imaging (denoted in blue) in the direction of the atrial. The resultant short-axis contours from the $\mathrm{RBF}$ morphing (denoted in red) is an estimate of the crosssectional area of the LA at that particular slice location.

\subsection{Projection/Morphing of short-axis contour (optional)}

This is an optional step in the event that the CMR imaging did not capture the entire geometry of the LA on the short-axis images (from above the mitral valve to the most basal LA slice - refer to Figure 1). The radial basis function $(\mathrm{RBF})$ method is then used for the iterative projection and morphing of the most basal short-axis contours in the atrial direction to the most basal LA slice.

RBFs are a popular means for interpolating scattered data for its ability to deal with irregular sets of data in multi-dimensional space in approximating high dimensional smooth surfaces [8].

The source points for the RBF are computed by the intersection of the most basal short-axis contours with the individual plane formed by the $2 \mathrm{CH}$ and $4 \mathrm{CH}$ long-axis contours respectively. The target points for the RBF are computed by the intersection of the $2 \mathrm{CH}$ and $4 \mathrm{CH}$ longaxis contours with the short-axis plane formed by the most basal short-axis contours projected in the LA direction by the spatial resolution of the out-of-plane CMR image. The resultant output from this step is an extrapolated short-axis contours that is an estimate of the cross-section of the LA at that particular slice location. 


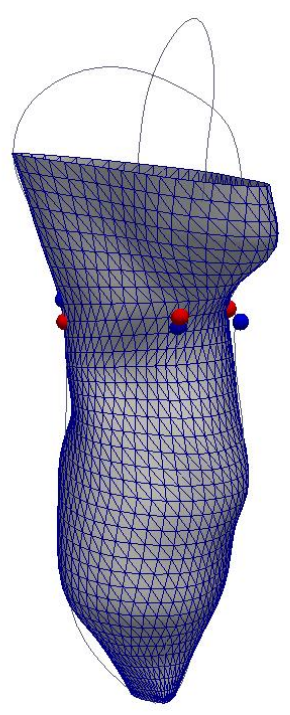

Figure 2. RBF morphing to reconstruct the non-planar LA-LV interface. The source points are denoted by the red spheres while the target points are denoted by the blue spheres. Refer to main text for detailed derivation of the source and target points.

\subsection{Morphing to reconstruct the LA-LV interface.}

The 3D LA-LV surface is then reconstructed from the short-axis contours into an unstructured triangular mesh using the approach described in our previous work [9]. To accurately calculate the LA and LV volumes, we first need to demarcate the non-planar interface between these two chambers. First, we identify the short-axis contours that is nearest to the LA-LV interface defined by the terminal points of the $\mathrm{LA}$ and $\mathrm{LV} 2 \mathrm{CH}$ and $4 \mathrm{CH}$ long axis contours. These 4 points will serve as the target points in the RBF. Next, we identify the source points for the RBF by intersection of the short-axis contours identified above with the individual plane formed by the $2 \mathrm{CH}$ and $4 \mathrm{CH}$ long-axis contours respectively. The resultant output from the morphing is a set of surface mesh with a non-planar LA-LV interface (refer to Figure 2).

\subsection{Reconstruction/Morphing of the planar basal surface.}

The 3D left heart model reconstructed up to this stage consists of the LA with an open planar surface (refer to Figure 2). We first construct a flat planar surface mesh by connecting all points from the segmented contours radially inwards to the centroid of the contours. Additional points on these radial lines are generated to generate an unstructured triangular mesh (refer to Figure 3).

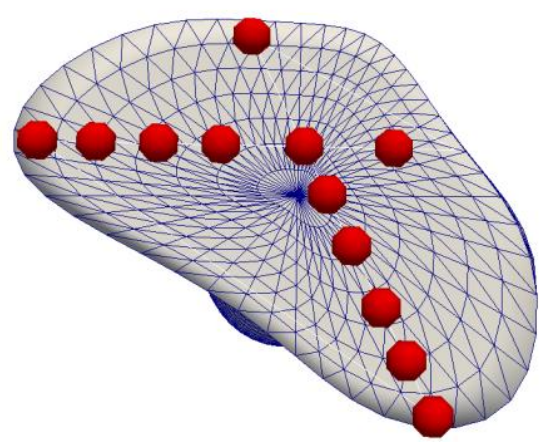

Figure 3. Flat planar surface mesh used for closing the LA basal surface. The source points used for morphing this planar surface to the geometry of the patientspecific LA patch is denoted by the red spheres.

To morph the LA patch into a hemisphere representing the closed surface of the LA, the information in the $2 \mathrm{CH}$ and $4 \mathrm{CH}$ long-axis contours representing the $\mathrm{LA}$ are utilized. First, the target points are computed by sampling these 2 contours individually along their arc length using a regular interval. Next, the source points are computed by projecting the $2 \mathrm{CH}$ and $4 \mathrm{CH}$ long-axis LA contours onto
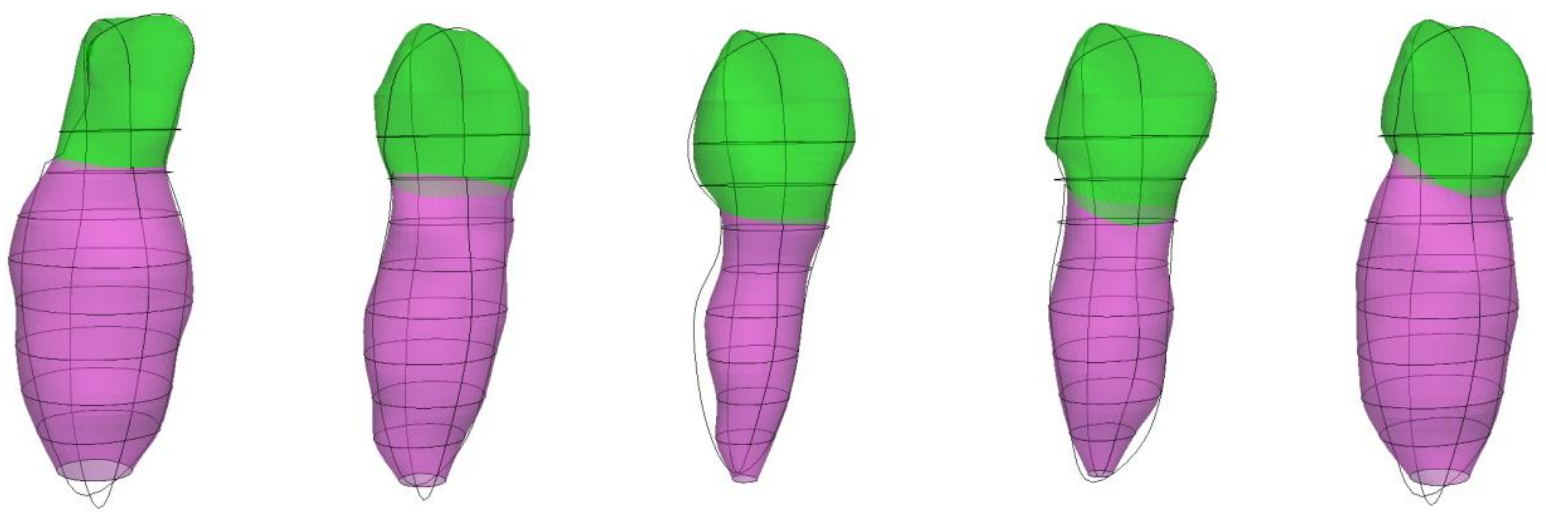

Figure 4. Selected instances of the LA (green) and LV (pink) geometries over the cardiac cycle for one subject. Enddiastole frame: 1st panel. End-systole frame: 3rd panel 
the flat planar surface and sampling the projected line using the same interval (refer to Figure 3 ). We also defined a minimum distance criterion between any pairs of source or targets points to ensure the numerical stability of the RBF. The resultant 3D geometry of the reconstructed LA and LV are shown in Figure 4 for selected instances of the cardiac cycle.

\section{Results and Conclusions}

The representation of the 3D LA and LV geometry in triangular meshes allow us to compute their respectively volumes and flow rate (time-derivative of the volume) as shown in Figure 5 for the normal controls, HF with preserved EF (HFPEF) and HF with reduced EF (HFREF) groups. Our approach allows us the ability to make comprehensive assessment of both systolic and diastolic parameters in the LV and LA for the clinical diagnosis of HF.

The limitation to our approach is the assumption that there is no misalignment between the short-axis contours and the long-axis contours representing the LA and LV. However, imaging artifacts arising from patient motion can potentially affect the accuracy of our approach.

In conclusion, we have described a geometry-based reconstruction algorithm for computing the LA and LV volumes automatically for the entire cardiac cycle by combining information from both the short- and long-axis from CMR imaging and demonstrated its potential clinical utility.
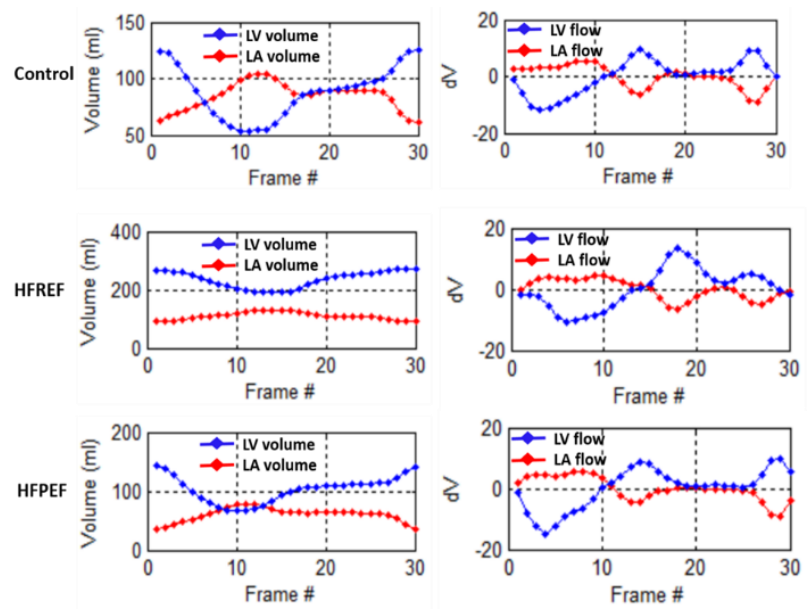

Figure 5. Volume (left panel) and flow rate (right panel) for the LA and LV computed using our approach.

\section{Acknowledgements}

This work was supported in part by research grant from the Agency for Science, Technology and Research (A*STAR), SERC Biomedical Engineering Programme
(BEP) Grant 1321480012 and National Medical Research Council (NMRC/OFIRG/0018/2016, Zhong).

\section{References}

[1] Pritchett AM, Mahoney DW, Jacobsen SJ, Rodeheffer RJ, Karon BL, Redfield MM. Diastolic dysfunction and left atrial volume: a population-based study. J Am Coll Cardiol. 2005;45:87-92.

[2] Rossi A, Cicoira M, Zanolla L, Sandrini R, Golia G, Zardini $\mathrm{P}$, et al. Determinants and prognostic value of left atrial volume in patients with dilated cardiomyopathy. J Am Coll Cardiol. 2002;40:1425.

[3] Dini FL, Cortigiani L, Baldini U, Boni A, Nuti R, Barsotti L, et al. Prognostic value of left atrial enlargement in patients with idiopathic dilated cardiomyopathy and ischemic cardiomyopathy. Am J Cardiol. 2002;89:518-23.

[4] Lang RM, Bierig M, Devereux RB, Flachskampf FA, Foster E, Pellikka PA, Picard MH, Roman MJ, Seward J, Shanewise JS, Solomon SD, Spencer KT, Sutton MS, Stewart WJ. Recommendations for chamber quantification: a report from the American Society of Echocardiography's Guidelines and Standards Committee and the Chamber Quantification Writing Group, developed in conjunction with the European Association of Echocardiography, a branch of the European Society of Cardiology. J Am Soc Echocardiogr. 2005;18(12):1440-63.

[5] Vizzardi E, D’Aloia A, Rocco E, Lupi L, Rovetta R, Quinzani F, Bontempi L, Curnis Md A, Dei Cas L. How should we measure left atrium size and function? J Clin Ultrasound. 2012;40(3):155-66.

[6] Hudsmith LE, Petersen SE, Francis JM, Robson MD, Neubauer S. Normal human left and right ventricular and left atrial dimensions using steady state free precession magnetic resonance imaging. $J$ Cardiovasc Magn Reson. 2005;7(5):775-82.

[7] Tobon-Gomez C, Geers A, Peters J, Weese J, Pinto K, Karim R, Schaeffter T, Razavi R, Rhode K. Benchmark for algorithms segmenting the left atrium from 3D CT and MRI datasets. IEEE Trans Med Imaging. 2015:34(7):1460-1473.

[8] M. J. D. Powell (1987), Radial basis functions for multivariate interpolation: A review. In Algorithms for Approximation, J. C. Mason and M. G. Cox, Eds. Clarendon Press.

[9] Zhao X, Tan RS, Tang HC, Teo SK, Su Y, Wan M, Leng S, Zhang JM, Allen J, Kassab GS, Zhong L. Left ventricular wall stress is sensitive marker of hypertrophic cardiomyopathy with preserved ejection fraction. Front Physiol. 2018; 9:250.

Address for correspondence.

Soo-Kng Teo.

1 Fusionopolis Way, \#16-16 Connexis, Singapore 138632 teosk@ihpc.a-star.edu.sg 